\title{
Genetic models for quantitatively inherited endosperm characters*
}

\author{
T. P. Bogyo, \\ R. C. M. Lance, $\dagger$ \\ Peggy Chevalier and \\ R. A. Nilan
}

\author{
Department of Agronomy and Soils, and Program in \\ Genetics and Cell Biology, Washington State \\ University, Pullman, Washington 99164-6420, U.S.A.
}

Genetic models are presented for the analysis of quantitatively inherited characters which are expressed in the triploid endosperm of plants. These models are appropriate for various populations derived from crosses between two homozygous parents where, as a consequence, in the reference population the gene frequencies are half at all segregating loci. The first model, which allows for the estimation of genotypic values, including additive, dominance and first order epistatic effects, can be used with data from the two parental lines, two reciprocal $F_{1}$ populations, the $F_{2}$ and the four backcross populations using the $F_{1}$ as one of the parents. The model was not extended to those four other backcrosses involving the $F_{1 R}$ populations as one of the parents. A second model is presented for the estimation of genetic variance components, including the variances of first order epistatic effects. A third model is described for the estimation of genetic variance components in nested designs which arise in pedigree breeding programs using self fertilization. Data from appropriate experiments are used to illustrate the use of the models.

\section{INTRODUCTION}

Mathematical models for the estimation of genotypic values of quantitatively inherited characters have been developed by Mather (1949), Mather and Jinks (1971), Kempthorne (1957), Cockerham $(1956,1963)$ and others. Most of these models were developed for populations of diploid organisms. An extension of these models to polyploid and haploid organisms was considered by Dessureaux (1963) and Mather and Jinks (1971), but without consideration of non-allelic interactions and only for cases where segregation is not affected by linkage or double reduction. A triploid model for the analysis of the quantitative genetics of $\alpha$-amylase in wheat endosperm was suggested by Gale (1976), but his model only involves main effects. In this paper we use a scale different from that of Gale (1976) and we also include interaction

\footnotetext{
* Scientific Paper No. 7678. College of Agriculture and Home Economics Research Center Washington State University, Pullman, Project No.'s 1006, 4006 and 7006. This investigation was supported in part by the Washington State University Foundation, The Washington State Nutritional Quality Evaluation Laboratory, The American Malting Barley Association, and the Great Western Malting Company.

$\dagger$ Present address: Dept. of Agronomy, Waite Agricultural Research Institute, The University of Adelaide, Glen Osmond, South Australia 5064.
}

components for characters expressed in triploid tissues, such as the endosperm of otherwise diploid plant individuals. We consider this to be an important extension of the theory because many of the economically valuable characters in plants express themselves in the endosperm of the seed; the model will therefore assist geneticists and plant breeders to estimate genetic parameters: both means and variances of these characters.

Throughout the paper we will make the following assumptions: (a) regular diploid and solely Mendelian inheritance, (b) no environmental correlations among relatives, (c) no linkages (d) the progenies or relatives are considered to be random members of their particular generation of self fertilization or crossing. When using models with interaction parameters included it is necessary to assume (e) that in segregating loci which affect the particular character studied, all alleles positively influencing the character are located in one of the parent inbreds, or, in other words using the terminology introduced by Mather and Jinks (1971), all the increasing alleles for the genes are associated in one of the parents. Although this paper offers an extension to the model of Mather and Jinks (1971), the notation of " $a$ " for additive and " $d$ " for dominance effects, as originally introduced by Fisher (1918), is used. 


\section{THE ANALYSIS OF MEANS}

\section{Main effect components: additive model with dominance}

The triploid endosperm of members of the grass family arises from the fusion of two identical gametes from the female parent with one gamete of the male parent. If we consider two alleles, $B$ and $b$, at a given locus, the endosperm can have one of four possible genotypes: $B B B, B B b, B b b$ and $b b b$. Four parameters will be necessary to describe the genotypic values of the above four genotypes. These are $m$, the mean, $a$ which measures the average effect of a single $B$ allele, $d_{1}$, measuring the deviation of the phenotype of the $B B b$ individual from its expected value without dominance, and $d_{2}$ measuring the deviation of the phenotype of the $B b b$ individuals from their expected value without dominance. Because the model assumes gene frequencies of 0.5 for all segregating loci the decomposition of the genotypic value as presented here is valid only for cases where the populations under study involve crosses between two pure lines $\left(P_{1}\right.$ and $\left.P_{2}\right)$, resulting in an $F_{1}$ population and where subsequent populations are obtained either by selfing or by backcrossing to either the parental or $F_{1}$ generations. The model will allow for $n$ loci and we restrict consideration to first order interactions of non-allelic gene-effects (epistasis).

The genetic effects are scaled as in fig. 1., in which $\mathbf{m}$ is the midpoint between the two triple homozygote parents $B B B$ and $b b b$, and all the other parameters are as described earlier. The midpoint should not be confused with $m$ which is the notation for the mean of the reference population. In the absence of dominance the value of the $B B B$ genotype would be $m+3 a / 2$, the values of $B B b$, $B b b$ and $b b b$ would respectively be $m+a / 2, m-$ $a / 2$ and $m-3 a / 2$. We need to consider two different cases of dominance, where the heterozygotes $B B b$ and $B b b$ deviate from their respective genotypic values under the assumption of "no dominance" by increments (or decrements) of $d_{1}$ and $d_{2}$. The actual values for $B B b$ and $B b b$ would, therefore be $m+a / 2+d_{1}$ and $m-a / 2+d_{2}$ respectively.

Using the two diploid parents $\mathrm{P}_{1}(B B)$ and $\mathrm{P}_{2}$ ( $b b$ ) one may produce two different $F_{1}$ populations depending on which parent is being used as a female parent. We shall designate the cross $P_{1} \times P_{2}$ as $F_{1}$, (by convention the first mentioned one is the female) and the reciprocal, $\mathrm{P}_{2} \times \mathrm{P}_{1}$ as $\mathrm{F}_{1 R}$, then for the $B-b$ locus the $F_{1}$ will have the genotype $B B b$ and the $F_{1 R}$ the genotype $B b b$ in the endosperm of the seeds produced by the respective female parent; it has to be remembered that the seed produced by any plant belongs to the next generation of populations. If $n$ loci affect a particular quantitative character, we define $[a]$ as $\sum a_{i}$ where $a$ is summed over all loci, $i$ taking all the values from 1 to $n$. Similarly $\left[d_{1}\right]$ and $\left[d_{2}\right]$ are designated as summations over all loci of the individual $d_{1}$ and $d_{2}$ values respectively. Thus the actual genotypic values of $F_{1}$ and $F_{1 R}$ endosperm will be $m+[a] / 2+\left[d_{1}\right]$ and $m-[a] / 2+\left[d_{2}\right]$ respectively.

In an $F_{2}$ population seed, which has been obtained by selfing either $F_{1}$ or $F_{\mathrm{r} R}$ plants, one would expect a segregation of $\frac{1}{4} B B B$ to $\frac{1}{4} B B b$ to $\frac{1}{4} B b b$ to $\frac{1}{4} b b b$ endosperm, considering the $B-b$ locus only. Summed over all loci the expected genotypic value of an $F_{2}$ plant will thus be $m+$ $\left[d_{1}\right] / 4+\left[d_{2}\right] / 4$, because there will be an equal number of positive and negative $[a]$ values canceling each other.

There are eight types of backcross generations possible in the endosperm; however we shall consider only those four which involve $F_{1}$ (and not $\mathrm{F}_{1 R}$ ). Each backcross generation will have different expected values. We designate the $F_{1} \times P_{1}$ cross as $\mathrm{BC}_{1}$, the $\mathrm{P}_{1} \times \mathrm{F}_{1}$ as $\mathrm{BC}_{1 R}$, the $\mathrm{F}_{1} \times \mathrm{P}_{2}$ as $\mathrm{BC}_{2}$ and the $\mathrm{P}_{2} \times \mathrm{F}_{1}$ as $\mathrm{BC}_{2 R}$. For the $B-b$ locus the endosperm of the $B C_{1}$ populations will on the average consist of $\frac{1}{2} B B B$ and $\frac{1}{2} B b b$ individuals with the following mean genotypic value:

$$
\frac{1}{2}\left[(m+3 a / 2)+\left(m-a / 2+d_{2}\right)\right]=m+a / 2+d_{2} / 2 \text {. }
$$

Similarly the means of the other three backcross populations will be $m+a+d_{1} / 2$ for the $\mathrm{BC}_{1 R}$,



Figure 1 Additive $(a)$ and dominance $\left(d_{1}\right)\left(d_{2}\right)$ genetic parameters. The four possible genotypes at a single locus $B-b$ in triploid tissue. Deviations are scales in respect to the midpoint $(m)$ between the two parents $\mathrm{P}_{1}$ and $\mathrm{P}_{2}$ and in respect to the additive effect $(a)$ in a single allele $B$. 
$m-a / 2+d_{1} / 2$ for the $\mathrm{BC}_{2}$ and $m-a+d_{2} / 2$ for the $\mathrm{BC}_{2 R}$.

An overall summary of the expected frequencies and population means for the $B-b$ locus and the expected mean genotypic values summing over all the loci is found in tables 1 and 3 .

Table 1 Relative frequencies of genotypes with respect to a locus. $B-b$ of triploid tissues in populations derived from a cross between two homozygous lines, considering main effects only

\begin{tabular}{lclll}
\hline $\begin{array}{l}\text { Genotype } \\
\text { Genotypic }\end{array}$ & $B B B$ & $B B b$ & $B b b$ & $b b b$ \\
value & $3 a / 2$ & $a / 2+d_{1}$ & $-a / 2+d_{2}$ & $-3 a / 2$ \\
\hline
\end{tabular}

$\begin{array}{lllll}\text { Population } & & & & \\ \mathrm{BC}_{1} & \frac{1}{2} & 0 & \frac{1}{2} & 0 \\ \mathrm{BC}_{1 R} & \frac{1}{2} & \frac{1}{2} & 0 & 0 \\ \mathrm{BC}_{2} & 0 & \frac{1}{2} & 0 & \frac{1}{2} \\ \mathrm{BC}_{2 R} & 0 & 0 & \frac{1}{2} & \frac{1}{2} \\ \mathrm{~F}_{1} & 0 & 1 & 0 & 0 \\ \mathrm{~F}_{1 R} & 0 & 0 & 1 & 0 \\ \mathrm{~F}_{2} & \frac{1}{4} & \frac{1}{4} & \frac{1}{4} & \frac{1}{4} \\ \mathrm{P}_{1} & 1 & 0 & 0 & 0 \\ \mathrm{P}_{2} & 0 & 0 & 0 & 1\end{array}$

Table 2 The coefficients of main and interaction effects for two loci, $B-b$, and $G-g$ in triploid tissues

\begin{tabular}{lcrrrrrrrrr}
\hline & $m$ & $a_{b}$ & $a_{g}$ & $d_{1 b}$ & $d_{1 g}$ & $d_{2 g}$ & $d_{2 g}$ & $a a$ & $a d$ & $d d$ \\
\hline BBBGGG & 1 & 1 & 1 & 0 & 0 & 0 & 0 & 1 & 0 & 0 \\
BBBGGg & 1 & 1 & 0 & 0 & 1 & 0 & 0 & 0 & 1 & 0 \\
BBBGgg & 1 & 1 & 0 & 0 & 0 & 0 & 1 & 0 & 1 & 0 \\
BBBggg & 1 & 1 & -1 & 0 & 0 & 0 & 0 & -1 & 0 & 0 \\
BBbGGG & 1 & 0 & 1 & 1 & 0 & 0 & 0 & 0 & 1 & 0 \\
BBbGGg & 1 & 0 & 0 & 1 & 1 & 0 & 0 & 0 & 0 & 1 \\
BBbGgg & 1 & 0 & 0 & 1 & 0 & 0 & 1 & 0 & 0 & 1 \\
BBbggg & 1 & 0 & -1 & 1 & 0 & 0 & 0 & 0 & -1 & 0 \\
BbbGGG & 1 & 0 & 1 & 0 & 0 & 1 & 0 & 0 & 1 & 0 \\
BbbGGg & 1 & 0 & 0 & 0 & 1 & 1 & 0 & 0 & 0 & 1 \\
BbbGgg & 1 & 0 & 0 & 0 & 0 & 1 & 1 & 0 & 0 & 1 \\
Bbbggg & 1 & 0 & -1 & 0 & 0 & 1 & 0 & 0 & -1 & 0 \\
bbbGGG & 1 & -1 & 1 & 0 & 0 & 0 & 0 & -1 & 0 & 0 \\
bbbGGg & 1 & -1 & 0 & 0 & 1 & 0 & 0 & 0 & -1 & 0 \\
bbbGgg & 1 & -1 & 0 & 0 & 0 & 0 & 1 & 0 & -1 & 0 \\
bbbggg & 1 & -1 & -1 & 0 & 0 & 0 & 0 & 1 & 0 & 0 \\
\hline
\end{tabular}

\section{Interaction components: model with epistasis}

The model may include non-allelic interaction (epistasis) components. Considering only two loci, $B-b$ and $G-g$, there are 16 possible triploid genotypes. These are listed, together with their genotypic components in table 2 . The columns in this table represent the mean $(m)$, the additive effects at each locus $\left(a_{b}\right.$ and $\left.a_{g}\right)$, the dominance
Table 3 Coefficients of expected genetic parameters for triploid tissues in populations derived from two homozygous lines

\begin{tabular}{llrlllll}
\hline \multicolumn{1}{c}{ Genetic parameter } & & & \\
Population & $m$ & {$[a]$} & {$\left[d_{1}\right]$} & {$\left[d_{2}\right]$} & {$[a a]$} & {$[a d]$} & {$[d d]$} \\
\hline $\mathrm{BC}_{1}$ & 1 & $\frac{1}{2}$ & 0 & $\frac{1}{2}$ & $\frac{1}{4}$ & $\frac{1}{2}$ & $\frac{1}{4}$ \\
$\mathrm{BC}_{1 R}$ & 1 & 1 & $\frac{1}{2}$ & 0 & $\frac{1}{4}$ & $\frac{1}{2}$ & $\frac{1}{4}$ \\
$\mathrm{BC}_{2}$ & 1 & $-\frac{1}{2}$ & $\frac{1}{2}$ & 0 & $\frac{1}{4}$ & $-\frac{1}{2}$ & $\frac{1}{4}$ \\
$\mathrm{BC}_{2 R}$ & 1 & -1 & 0 & $\frac{1}{2}$ & $\frac{1}{4}$ & $-\frac{1}{2}$ & $\frac{1}{4}$ \\
$\mathrm{~F}_{1}$ & 1 & $\frac{1}{2}$ & 1 & 0 & 0 & 0 & 1 \\
$\mathrm{~F}_{1 R}$ & 1 & $-\frac{1}{2}$ & 0 & 1 & 0 & 0 & 1 \\
$\mathrm{~F}_{2}$ & 1 & 0 & $\frac{1}{4}$ & $\frac{1}{4}$ & 0 & 0 & $\frac{1}{4}$ \\
$\mathrm{P}_{1}$ & 1 & $\frac{3}{2}$ & 0 & 0 & 1 & 0 & 0 \\
$\mathrm{P}_{2}$ & 1 & $-\frac{3}{2}$ & 0 & 0 & 1 & 0 & 0 \\
\hline
\end{tabular}

effects $\left(d_{1 b}, d_{1 g}, d_{2 b}\right.$ and $\left.d_{2 g}\right)$, the combined additive by additive $(a a)$, additive by dominance $(a d)$ and dominance by dominance $(d d)$ interactions. These can further be grouped representing the genotypic value $(G)$ as

$$
G=m+[a]+\left[d_{1}\right]+\left[d_{2}\right]+[a a]+[a d]+[d d]
$$

where the square brackets of the interaction components mean sums of similar effects over all $n(n-$ 1) $/ 2$ possible pairs of loci.

We shall consider only the nine populations described before: $\mathrm{P}_{1}, \mathrm{P}_{2}, \mathrm{~F}_{1}, \mathrm{~F}_{1 R}, \mathrm{~F}_{2}$ and the four different backcross ones. The expected values for these are listed in table 3 . The reader should be reminded, that the additive value is not scaled from the midpoint as in Mather and Jinks (1971). Therefore the interaction components cannot be derived simply by multiplying the coefficients of the main effect parameters, as can be done for a diploid model. Also dominance effects are deviations from the additive effects (under the assumption of no dominance) rather than deviations from the midpoint. In fact the midpoint in this model does not serve much useful purpose other than a comparison with a diploid model.

The seven parameters of the model can be estimated from the nine observed means by the equating the observed values with the expected ones. Thus a mean value for the observed character for each of the nine populations is available together with their variances.

It is, of course, customary to first test a model without epistasis, in this case using only the first four columns of the $X$ matrix. A significant departure from a good fit as measured by the $\chi^{2}$ test, would lead to further test, involving one or more of the epistasis parameters of the $X$ matrix. It should be pointed out that occasionally a good fit of the "main effects only" model can be obtained by a suitable transformation. Before continuing to 
the extended model, in case of a significant chisquare value it is prudent to carry out the same test on the transformed data. It should also be remembered that the $[a]$ and $\left[d_{i}\right]$ are sums of the signed $a_{i}$ and $d_{i}$ over all the loci, and these may differ greatly from locus to locus.

The estimation procedure is that of the standard weighted least squares as described by Mather and Jinks (1971).

\section{THE ANALYSIS OF VARIANCES}

Since we are dealing here with a discrete distribution, the variance of the different populations can be derived from

$$
\sum p_{i} X_{i}^{2}-\left(\sum p_{i} X_{i}\right)^{2}
$$

where $p_{i}$ refer to the relative proportion of a particular genotype in the population, and $X_{i}$, in this case, to the appropriate genotypic components of those genotypes (as in table 1). We will only consider the main effect components $a$, and $d_{i}$. As an example for the $\mathrm{BC}_{1}$ generation the population will consist of $\frac{1}{2} B B B$ and $\frac{1}{2} B b b$ endosperm with genotypic values of $3 a / 2$ and $-a / 2+d_{2}$ respectively, with the mean of the population being $a / 2+$ $d_{2} / 2$. Therefore the genotypic variance, $V_{(\mathrm{BC})}$, can be calculated as

$$
\begin{aligned}
V_{(\mathrm{BCl})} & =\frac{1}{2}(3 a / 2)^{2}+\frac{1}{2}\left(-a / 2+d_{2}\right)^{2}-\left(a / 2+d_{2} / 2\right)^{2} \\
& =a^{2}+\frac{1}{4} d_{2}^{2}-a d_{2} .
\end{aligned}
$$

The genotypic components of the variance for the populations $\mathrm{BC}_{1 R}, \mathrm{BC}_{2}, \mathrm{BC}_{2 R}$ and $\mathrm{F}_{2}$ can be calculated in a similar manner.

We define $A=\sum a_{i}^{2}, D_{1}=\sum d_{1 i}^{2}, \quad D_{2}=\sum d_{2 i}^{2}$, $A D_{1}=\sum a_{i} d_{1 i}, A D_{2}=\sum a_{i} d_{2 i}$ and $D_{1} D_{2}=\sum d_{1 i} d_{2 i}$. Using these we can now construct the expected variance components for triploid systems as in table 4. The method of estimation is that of Hayman (1960).

Table 4 Coefficients of expected genetic variance components for triploid tissues in populations derived from two homozygous lines

\begin{tabular}{lcccccc}
\hline \multicolumn{7}{c}{ Genetic variance components } \\
Population & $A$ & $D_{1}$ & $D_{2}$ & $A D_{1}$ & $A D_{2}$ & $D_{1} D_{2}$ \\
\hline $\mathrm{BC}_{1}$ & 1 & 0 & $\frac{1}{4}$ & 0 & -1 & 0 \\
$\mathrm{BC}_{1 R}$ & $\frac{1}{4}$ & $\frac{1}{4}$ & 0 & $-\frac{1}{2}$ & 0 & 0 \\
$\mathrm{BC}_{2}$ & 1 & $\frac{1}{4}$ & 0 & 1 & 0 & 0 \\
$\mathrm{BC}_{2 R}$ & $\frac{1}{4}$ & 0 & $\frac{1}{4}$ & 0 & $\frac{1}{2}$ & 0 \\
$\mathrm{~F}_{2}$ & $\frac{5}{4}$ & $\frac{3}{16}$ & $\frac{3}{16}$ & $\frac{1}{4}$ & $-\frac{1}{4}$ & $-\frac{1}{8}$ \\
\hline
\end{tabular}

\section{Nested designs of self fertilisation}

Inbred populations derived from $F_{2}$ individual plants by selfing can be subdivided into lines following a nested scheme. This introduces various additional observational components of variance. Without dominance the simplest way to represent this situation can be represented by the equation

$$
V_{T}=V_{b}+V_{w}
$$

where $V_{T}$ is the total genetic variance and equals $(1+F) V_{A}, V_{b}$ is the between lines component and equals $2 F V_{A}$, and $V_{w}$ is the within lines component and equals $(1-F) V_{a}$, where $V_{A}$ is the additive genetic variance and $F$ is the coefficient of inbreeding. Of course when lines are completely inbred, $(F=1)$, all genetic variation comes from the variation among lines and none from within lines.

If pedigrees are maintained during the course of inbreeding it can also be seen that various degrees of sublines can be defined according to which common ancestor one refers to as the originator of the subline. (See fig. 2.). In the $F_{3}$ generation (since the only ancestors one may refer to must come from the noninbred $\mathrm{F}_{2}$ generation) there is only one kind of line possible: that which originates with the parent $F_{2}$, and, as a consequence, there are only two kinds of possible variances: those calculated from $\mathrm{F}_{3}$ family means and the average "within families" mean. We shall follow the notation of Mather and Jinks (1971). In their notation $V_{1 F 3}$ is the among families variance and $V_{2 F 3}$ the within families variance. There is only one possible parent-offspring covariance i.e., the covariance between $\mathrm{F}_{2}$ individuals and the family means of their own offspring, denoted by $W_{1 F 23}$.

In the $\mathrm{F}_{4}$ generation it is possible to discern between two kinds of sublines, those that can be traced back to a single $F_{2}$ grandparent, the variance of the means of these lines is denoted as $V_{1 F 4}$, and those that go back only to the $F_{3}$ parents; the variance of the means of these lines are denoted as $V_{2 F 4}$. The average within families variance is now denoted as $V_{3 F 4}$. There are now two kinds of covariances possible: those between $F_{3}$ family means and $\mathrm{F}_{4}$ "group" means, where a group is a set of $\mathrm{F}_{4}$ plants originating from the same $\mathrm{F}_{2}$ grandparent, denoted as $W_{1 F 34}$, and that between $F_{3}$ individuals and the means of their offspring families in the $\mathrm{F}_{4}$ generation, these are denoted as $W_{2 F 34}$. In a similar manner in the $F_{5}$ generation one could calculate four types of variances and three different covariances, in the $\mathrm{F}_{6}$ five variances and four covariances etc. In this paper we shall 


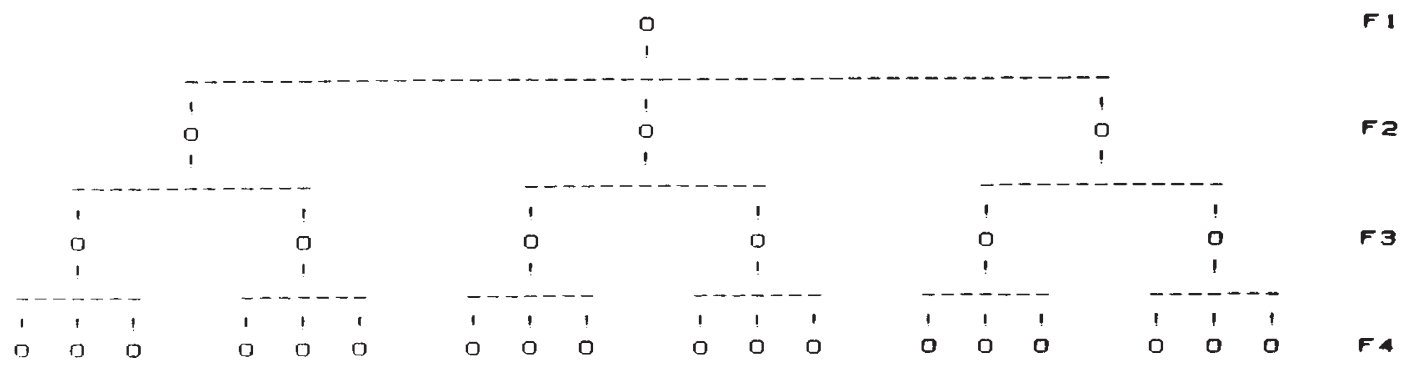

Figure 2 Diagrammatic representation of a nested design arising from selfing. Individuals are represented by 0's, all individuals under a broken line are families.

develop these variances only up to the $\mathrm{F}_{4}$ generation. The covariances can be calculated from the formula

$$
\sum \sum p_{i j} X_{i} Y_{j}-\sum p_{i} X_{i} \sum p_{j} Y_{j}
$$

where $p_{i j}$ is the joint probability of the occurrence of $X_{i}$ with $Y_{j}$ and $X_{i}$ and $Y_{j}$ are (in this case) the appropriate genotypic values. These same formulae can, of course, be used to calculate variances with $X_{i}=Y_{j}$.

We shall first calculate $V_{1 F 3}$, the variance of $\mathrm{F}_{3}$ family means which come from individual $\mathrm{F}_{2}$ plants. The expected proportion of the genotypes will be $\frac{1}{4}$ each of $B B B, B B b, B b b$ and $b b b$. The mean genotypic value of these populations is expected to be $\left(d_{1}+d_{2}\right) / 8$. Therefore:

$$
\begin{aligned}
V_{1 F 3}= & \frac{1}{4}(3 a / 2)^{2}+\frac{1}{2}\left(d_{1} / 4+d_{2} / 4\right)^{2} \\
& +\frac{1}{4}(-3 a / 2)^{2}-\left(d_{1} / 8+d_{2} / 8\right)^{2} \\
= & \frac{9}{8} a^{2}+\frac{1}{64} d_{1}^{2}+\frac{1}{64} d_{2}^{2}-\frac{1}{32} d_{1} d_{2} .
\end{aligned}
$$

The within family variance, $V_{2 F 3}$ is expected to be $(1 / 2)\left(V_{F 2}\right)$, thus:

$$
V_{2 F 3}=\frac{5}{8} a^{2}+\frac{3}{32} d_{1}^{2}+\frac{3}{32} d_{2}^{2}-\frac{1}{8} a d_{1}-\frac{1}{8} a d_{2}-\frac{1}{16} d_{1} d_{2} .
$$

The covariance between the means of $F_{3}$ families and their $F_{2}$ parents, $W_{1 F 23}$, is calculated as follows:

$$
\begin{aligned}
W_{1 F 23}= & \frac{1}{4}(3 a / 2)^{2}+\frac{1}{4}\left(a / 2-d_{1}\right)\left(d_{1} / 4+d_{2} / 4\right) \\
& +\frac{1}{4}(-a / 2+d)\left(d_{1} / 4+d_{2} / 4\right) \\
& +\frac{1}{4}(-3 a / 2)^{2}-\left(d_{1} / 4+d_{2} / 4\right)\left(d_{1} / 8+d_{2} / 8\right) \\
= & \frac{9}{8} a^{2}+\frac{1}{32} d_{1}^{2}+\frac{1}{32} d_{2}^{2}+1 / 16 d_{1} d_{2} .
\end{aligned}
$$

Similar derivations can be obtained for $V_{1 F 4}$, $V_{2 F 4}, V_{3 F 4}, W_{1 F 34}$ and $W_{2 F 34}$. Summing over all loci the expectations of genetic variance components can be obtained, these are listed in table 5 .
Table 5 Coefficients of expected genetic variance components under selfing for triploid tissues

\begin{tabular}{ccccccc}
\hline $\begin{array}{l}\text { Variance or } \\
\text { Covariance }\end{array}$ & $A$ & $D_{1}$ & $D_{2}$ & $A D_{1}$ & $A D_{2}$ & $D_{1} D_{2}$ \\
\hline $\mathrm{F}_{2}: V_{1 F 2}$ & $\frac{5}{4}$ & $\frac{3}{16}$ & $\frac{3}{16}$ & $\frac{1}{4}$ & $-\frac{1}{4}$ & $-\frac{1}{8}$ \\
$\mathrm{~F}_{3}: V_{1 F 3}$ & $\frac{9}{8}$ & $\frac{1}{64}$ & $\frac{1}{64}$ & 0 & 0 & $\frac{1}{32}$ \\
$V_{2 F 3}$ & $\frac{5}{8}$ & $\frac{3}{32}$ & $\frac{3}{32}$ & $\frac{1}{8}$ & $-\frac{1}{8}$ & $-\frac{1}{16}$ \\
$W_{1 F 23}$ & $\frac{9}{8}$ & $\frac{1}{32}$ & $\frac{1}{32}$ & 0 & 0 & $\frac{1}{16}$ \\
$\mathrm{~F}_{4}: V_{1 F 4}$ & $\frac{9}{8}$ & $\frac{1}{256}$ & $\frac{1}{256}$ & 0 & 0 & $\frac{1}{128}$ \\
$V_{2 F 4}$ & $\frac{9}{16}$ & $\frac{1}{128}$ & $\frac{1}{128}$ & 0 & 0 & $\frac{1}{64}$ \\
$V_{3 F 4}$ & $\frac{5}{16}$ & $\frac{3}{64}$ & $\frac{3}{64}$ & $\frac{1}{16}$ & $-\frac{1}{16}$ & $-\frac{1}{32}$ \\
$W_{1 F 34}$ & $\frac{9}{8}$ & $\frac{1}{128}$ & $\frac{1}{128}$ & 0 & 0 & $\frac{1}{64}$ \\
$W_{2 F 34}$ & $\frac{9}{16}$ & $\frac{1}{64}$ & $\frac{1}{64}$ & 0 & 0 & $\frac{1}{32}$ \\
\hline
\end{tabular}

\section{FITTING DATA TO THE MODELS}

The following data come from Lance (1984). Two homozygous lines of six-rowed barley (Hordeum vulgare L.), one with high the other with low $\beta$-glucan content were used to obtain the nine different populations. They were derived from crosses involving $P_{1}, P_{2}$ and $F_{1}$ generations, as described earlier in this paper. $\beta$-glucan is a chemical compound which occurs in the triploid endosperm tissue of the barley seed, which in turn belongs to the progeny generation of the mother plant. Replicated lots of seeds were assayed for total $\beta$-glucan content, the means expressed as percent of dry weight and adjusted for daily variations in the assaying process, together with their variances are listed in table 6 . The table also contains the number of seeds assayed in each population. The difference in average $\beta$-glucan percentage between the two parent lines was 2.213 per cent which is more than half of the average percentage of the low $\left(\mathrm{P}_{2}\right)$ line. The mean percentage of all the other populations was intermediate between the two parent lines. Both parent lines had significantly different means from any other population in the study. Only the two (reciprocal) 
Table 6 Average $\beta$-glucan percentage values for the nine experimental populations together with the variances of these means and the sizes of the samples. Note: Two means followed by the same letters are not significantly different at the $5 \%$ level

\begin{tabular}{llll}
\hline Population & Mean & $\begin{array}{l}\text { Variance } \\
\text { of mean }\end{array}$ & $\begin{array}{l}\text { Sample } \\
\text { size }\end{array}$ \\
\hline $\mathrm{BC}_{1}$ & $5 \cdot 657 b$ & 0.0036 & 60 \\
$\mathrm{BC}_{1 R}$ & $5 \cdot 500 b$ & 0.0039 & 60 \\
$\mathrm{BC}_{2}$ & $4 \cdot 593 f$ & 0.0036 & 59 \\
$\mathrm{BC}_{2 R}$ & $4 \cdot 826 e$ & 0.0037 & 59 \\
$\mathrm{~F}_{1}$ & $5 \cdot 143 c d$ & 0.0037 & 60 \\
$\mathrm{~F}_{1 R}$ & $5 \cdot 308 c$ & 0.0035 & 60 \\
$\mathrm{~F}_{2}$ & $5 \cdot 129 d$ & 0.0015 & 160 \\
$\mathrm{P}_{1}$ & $6 \cdot 344 a$ & 0.0055 & 39 \\
$\mathrm{P}_{2}$ & $4 \cdot 131 g$ & $0 \cdot 0060$ & 40 \\
\hline
\end{tabular}

$F_{1}$ generations, the $F_{1}$ and $F_{2}$ generations and the $\mathrm{BC}_{1}$ and $\mathrm{BC}_{1 R}$ had means which did not differ significantly. The estimates of the genotypic effects for both the four parameter (main effects only) and the seven parameter models (main effects and interactions) are listed in table 7 . The main effects

Table 7 Estimates of the genetic parameters of $\beta$-glucan percentage in barley together with their standard errors using the triploid model

\begin{tabular}{|c|c|c|}
\hline $\begin{array}{l}\text { Estimated } \\
\text { parameter }(\hat{b})\end{array}$ & $\begin{array}{l}\text { Four parameter } \\
\text { model }\end{array}$ & $\begin{array}{l}\text { Seven parameter } \\
\text { model }\end{array}$ \\
\hline $\begin{array}{l}m \\
{[a]} \\
{\left[d_{1}\right]} \\
{\left[d_{2}\right]} \\
{[a a]} \\
{[a d]} \\
{[d d]}\end{array}$ & $\begin{array}{r}5 \cdot 161 \pm 0.041 \\
0.670 \pm 0.077 \\
-0 \cdot 442 \pm 0.077 \\
0.466 \pm 0.077\end{array}$ & $\begin{array}{c}5 \cdot 178 \pm 0.203^{*} \\
0.738 \pm 0.036^{*} \\
-0.731 \pm 0.507 \\
0.246 \pm 0.507 \\
0.059 \pm 0.196 \\
-0.236 \pm 0.081^{*} \\
0.289 \pm 0.318 \\
\chi^{2} 2 \mathrm{df}=2.71\end{array}$ \\
\hline
\end{tabular}

* Denotes the estimate to be significantly different from zero using $z$ statistics.

only model was fitted first, giving a significant $\left(\chi^{2}=16 \cdot 43,5 \mathrm{df}\right)$ lack of fit at the 5 per cent level of significance. Various transformations did not improve the fit. The seven parameter model, on the other hand, gave an excellent fit $\left(\chi^{2}=2 \cdot 71\right.$, $2 \mathrm{df})$. of the parameters estimated the additive effect $[a]$ and the additive by dominance $[a d]$ effect were significantly different from zero at the 5 per cent level of significance. To calculate the significance the $z$ values were calculated as $(O-$ $E) / S E$, where $O$ is the observed value, $E$ the expected one and $S E$ the standard error of the observed parameter.
Table 8 lists the estimates of the variance components together with their standard errors. The standard errors of the estimates (as is usual in these experiments) are too large, except for $E$, the environmental variance. It is somewhat reassuring

Table 8 Genetic variance component estimates from the nine experimental populations together with the standard errors of these estimates (obtained as the square roots of the diagonal elements of the variance-covariance matrix $\left(Z^{\prime} Z\right)^{-1} s^{2}$, and where $s^{2}=y^{\prime} y-v^{\prime} X^{\prime} y$

\begin{tabular}{lrl}
\hline Variance component & Estimate & $\begin{array}{l}\text { Standard error } \\
\text { of estimate }\end{array}$ \\
\cline { 1 - 2 }$A$ & 0.1434 & 0.523 \\
$D_{1}$ & 0.0208 & 1.368 \\
$D_{2}$ & -0.1006 & 1.369 \\
$A D_{1}$ & -0.0986 & 0.296 \\
$A D_{2}$ & 0.0179 & 0.295 \\
$E$ & 0.1372 & 0.071 \\
\hline
\end{tabular}

that the two largest variances are the additive and the environmental ones. However, the absolute values of the negatively estimated variance components are somewhat too large for comfort. This makes the estimation or heritabilities from the nine populations somewhat difficult. Leaving the negative values intact would make the estimates of heritability larger than $1(1 \cdot 19)$. Assuming that the negative values indicate zero variances the heritability (in the narrow sense) would be $0 \cdot 45$. Equating all non-additive variances with zero (since the non-negative ones are very small) will yield a heritability estimate of $0 \cdot 51$. Using $F_{3}$ and $F_{4}$ data, and calculating ${ }_{\mathrm{h}}{ }^{2}$ by using regression of $\mathrm{F}_{4}$ family means on parent $F_{3}$ family means will yield a regression coefficient of 0.73 , which, being the regression of offspring on midparent is a direct estimate of $h^{2}$. Using the phenotypic variance estimates of the $\mathrm{F}_{3}\left(V_{1 F 3}\right)$ and $\mathrm{F}_{4}\left(V_{2 F 4}\right)$, calculating the phenotypic covariance estimates from the regression $\left(W_{1 F 34}\right)$, and combining all the nonadditive components into a single value, the estimate of the additive genetic variance $[a]$ is $0 \cdot 1216$, not very different from the estimate from the nine other experimental populations. From the same data the environmental variance estimate is 0.0441 . Using these values heritability from the $F_{3}$ population is estimated to be 0.65 and from the $F_{4}$ data $0 \cdot 73$. Commenting on the four different estimates of heritability (ranging from 0.45 to 0.73 ) one could conclude that there is strong evidence for a reasonably high heritability of glucan percentage in barley seed. 


\section{REFERENCES}

COCKERHAM, C. C. 1956. Analysis of quantitative gene action. Brookhaven Symposia on Biology, 9, 56-68.

COCKERHAM, C. C. 1963. Estimation of genetic variances. In Hanson, W. K. and Robinson, H. F. (eds.), Statistical Genetics and Plant Breeding, National Academy of Sciences-National Research Council, Washington D.C., pp. 5394.

DESSUREAUX, L. 1963. Polyploids and polygenes. In Hanson, W. K. and Robinson, H. F. (eds.), Statistical Genetics and Plant Breeding, National Academy of Sciences-National Research Council, Washington D.C., 522 pp.

FISHER, R. A. 1918. The correlation between relatives on the supposition of Mendelian inheritance. Trans. Roy. Soc. Edinburgh, 52, 399-433.
GALE, M. D. 1976. High $\alpha$-amylase breeding and genetical aspects of the problem. Cereal Res. Commun., 4, 231-243.

HAYMAN, B. I. 1960. Maximum likelihood estimation of genetic components of variation. Biometrics, 16, 369-81.

KEMPTHORNE, O. 1957. An Introduction to Genetic Statistics. John Wiley \& Sons, New York, 545 pp.

LANCE, R. C. M. 1984. Genetic Studies of the $\beta$-glucan Content of Barley. Ph.D. thesis-Washington State University, Pullman, Washington 99163.

MATHER, K. 1949. Biometrical Genetics. Methuen, London, 162 $\mathrm{pp}$.

MATHER, K. AND JINkS, J. L. 1971. Biometrical Genetics. Cornell University Press, Ithaca, N.Y., 382 pp. 